OPEN ACCESS

Edited by:

Antonio Faciola,

University of Nevada, Reno,

United States

Reviewed by:

Sergio Uzzau,

University of Sassari, Italy

Jiangchao Zhao,

University of Arkansas, United States

*Correspondence: Xiurong Su suxiurong_public@163.com

Specialty section:

This article was submitted to

Systems Microbiology,

a section of the journal

Frontiers in Microbiology

Received: 28 January 2017

Accepted: 03 May 2017

Published: 17 May 2017

Citation:

Lu C, Sun T, Li Y, Zhang D, Zhou J and SuX (2017) Modulation of the Gut Microbiota by Krill Oil in Mice Fed a

High-Sugar High-Fat Diet.

Front. Microbiol. 8:905

doi: 10.3389/fmicb.2017.00905

\section{Modulation of the Gut Microbiota by Krill Oil in Mice Fed a High-Sugar High-Fat Diet}

\author{
Chenyang Lu ${ }^{1}$, Tingting Sun ${ }^{1}$, Yanyan $\mathrm{Li}^{2}$, Dijun Zhang ${ }^{1}$, Jun Zhou ${ }^{1}$ and Xiurong Su ${ }^{1 *}$ \\ ${ }^{1}$ School of Marine Science, Ningbo University, Ningbo City, China, ${ }^{2}$ Department of Food Science, Cornell University, \\ New York, NY, United States
}

Multiple lines of evidence suggest that the gut microbiota plays vital roles in metabolic diseases such as hyperlipidemia. Previous studies have confirmed that krill oil can alleviate hyperlipidemia, but the underlying mechanism remains unclear. To discern whether krill oil changes the structure of the gut microbiota during the hyperlipidemia treatment, 72 mice were acclimatized with a standard chow diet for 2 weeks and then randomly allocated to receive a standard chow diet (control group, $n=12$ ) or a high-sugar-high-fat (HSHF) diet supplemented with a low $(100 \mu \mathrm{g} / \mathrm{g} \cdot \mathrm{d}$, HSHF+LD group, $n=12)$, moderate (200 $\mu \mathrm{g} / \mathrm{g} \cdot \mathrm{d}$, HSHF+MD group, $n=12)$ or high dosage of krill oil $(600 \mu \mathrm{g} / \mathrm{g} \cdot \mathrm{d}$, HSHF+HD group, $n=12)$, simvastatin (HSHF+S group, $n=12)$ or saline (HSHF group, $n=12$ ) continuously for 12 weeks. The resulting weight gains were attenuated, the liver index and the low-density lipoprotein, total cholesterol and triglyceride concentrations showed a stepwise reduction in the treated groups compared with those of the control group. A dose-dependent modulation of the gut microbiota was observed after treatment with krill oil. Low- and moderate- doses of krill oil increased the similarity between the composition of the HSHF diet-induced gut microbiota and that of the control, whereas the mice fed the high-dose exhibited a unique gut microbiota structure that was different from that of the control and HSHF groups. Sixty-five key operational taxonomic units (OTUs) that responded to the krill oil treatment were identified using redundancy analysis, of which 26 OTUs were increased and 39 OTUs were decreased compared with those of the HSHF group. In conclusion, the results obtained in this study suggest that the structural alterations in the gut microbiota induced by krill oil treatment were dose-dependent and associated with the alleviation of hyperlipidemia. Additionally, the high-dose krill oil treatment showed combined effects on the alleviation of hyperlipidemia and obesity.

Keywords: krill oil, hyperlipidemia, gut microbiota, dose-dependent, mouse

\section{INTRODUCTION}

Humans face various metabolic diseases, including obesity, hyperlipidemia, hyperglycemia, and hypertension (Eckel et al., 2005). Hyperlipidemia comprises a group of plasma lipoprotein abnormalities, including increased triglycerides (TG), total cholesterol (TC), low density lipoprotein cholesterol (LDL), and reduced high density lipoprotein cholesterol (HDL) 
concentrations (Chen et al., 2014b). Hyperlipidemia is a dominant risk factor that contributes to cardiovascular disease (Abliz et al., 2014; Kim et al., 2015), and its development has become a difficult public health problem (Chen et al., 2014b). At present, drug therapy is the preferred treatment for hyperlipidemia. Various drugs have been used clinically, including simvastatin (Farnier and Davignon, 1998), acipimox (Shih et al., 1997), and clofibrate (Grundy et al., 1972). Generally, drug therapy is not regarded as an optimal long-term therapy due to its relatively high costs and adverse side effects. Therefore, intervention strategies are needed for the treatment of hyperlipidemia.

Accumulating evidence suggests that a disrupted balance between the host and the gut microbiota plays a vital role in lipid metabolism and the development of hyperlipidemia (Ley et al., 2006; Turnbaugh et al., 2006). Probiotic feeding reduced the development of a fat-mass in mice fed a high-fat diet, with a corresponding decrease in the phylum Firmicutes, increase in the phylum Bacteroidetes, and changes in 102 distinct taxa in the gut microbiota (Everard et al., 2011). In the hyperlipidemic mouse model, Lactobacillus administration led to a significant reduction in the serum lipids, an increased abundance of phylum Bacteroidetes and Verrucomicrobia and a reduced proportion of the phylum Firmicutes (Chen et al., 2014a). In the zebrafish model, Lactobacillus rhamnosus decreased the lipid content by increasing the ratio of Firmicutes, decreasing the abundance of Actinobacteria, and down-regulating the genes involved in cholesterol and triglycerides metabolism (Falcinelli et al., 2015). Furthermore, the addition of green tea powder promoted the growth of Lactobacillus in the intestine and strongly decreased the hepatic triacylglycerol and cholesterol accumulation and the amount of Akkermansia in the small intestine in mice fed a high fat diet (Axling et al., 2012). Some Chinese medicines have shown positive effects on hyperlipidemia control via modulation of the gut microbiota. The abundance of Sporobacter termitidis and Akkermansia muciniphila were increased significantly, whereas the proportions of Escherichia coli and Parabacteroides distasonis were reduced after treatment of hyperlipidemic B6 mice with Rhizoma coptidis alkaloids (He et al., 2016). The berberine supplement enriched short chain fatty acid (SCFA) - producing bacteria and reduced microbial diversity, which might contribute to the beneficial effects on dyslipidemia and hypercholesterolemia treatment (Kong et al., 2004; Zhang et al., 2015). In conclusion, the changes in the gut microbiota play an important role in the regulation of lipid metabolism and the alleviation of hyperlipidemia, and may have potential as a new hyperlipidemia treatment strategy.

Krill oil is extracted from Euphausia superb and contains eicosapentaenoic acid (EPA) and docosahexaenoic acid (DHA; Maki et al., 2009). The fatty acids in krill oil are present as phospholipids, which are absorbed well by the intestine compared with other triglycerides oils of marine origin (Harris et al., 1988; Araujo et al., 2014). Previous studies suggested that krill oil supplementation alleviated hyperlipidemia. For example, krill oil treatment suppressed lipid synthesis, up-regulated the genes involved in lipid oxidation, and down-regulated genes in lipogenesis (Burri et al., 2011; Ferramosca et al., 2012). Krill oil supplementation reduced the TG and TC levels and alleviated the hepatomegaly and hepatic steatosis induced by a high-fat diet (Tandy et al., 2009). Another study found that the hyperplasia and total histology score were dramatically decreased and that the serum cytokines levels remained unchanged after krill oil treatment (Ierna et al., 2010). In addition to a high concentration of omega-3 fatty acids, krill oil also contains astaxanthin, which is responsible for the deep red color (Figure S1). Astaxanthin is a strong lipid-soluble antioxidant that not only protects krill oil from oxidation (Hussein et al., 2007; Ikeuchi et al., 2007) but also provides additional health-promoting properties related to inflammation and hyperlipidemia (Bunea et al., 2004; Deutsch, 2007). At present, we have confirmed the benefits of krill oil in human health. However, there is a lack of evidence concerning whether krill oil alleviates hyperlipidemia via modulation of the gut microbiotan, and the dose-dependent effects of krill oil on hyperlipidemia remains unclear.

In this study, a randomized approach was used to assess the effect of krill oil on hyperlipidemia in mice. The biochemical blood indices, liver index, and shift in the structure of the gut microbiota in response to krill oil treatment at different doses were examined in mice. Our results provide new insights into the roles of the gut microbiota following treatment with different dose of krill oil for hyperlipidemia. We propose that this study will aid in the screening of intervention strategies for the amelioration of hyperlipidemia.

\section{MATERIALS AND METHODS}

\section{Experimental Design}

The experimental and animal care procedures were performed in accordance with the guidelines prepared by the Ningbo University Laboratory Animal Center (affiliated with the Zhejiang Laboratory Animal Common Service Platform, Ningbo, China). All protocols were approved by the Ningbo University Laboratory Animal Center under permit number SYXK (ZHE 2008-0110).

Seventy-two 10-week-old ICR mice $(22.1 \pm 2.6 \mathrm{~g}$, male, purchased from Laboratory Animal Center of Zhejiang province, SCXK (Zhejiang) 2014-0001, No. 1605200003) were randomly divided into six groups (12 mice per group) after 2 weeks of acclimatization with a standard chow diet. The twelve mice in each group were divided into three cages (four mice per cage). The mice in the control group were fed a standard chow diet (purchased from the Laboratory Animal Center of Ningbo University, Ningbo, China). The mice in the high-sugar high-fat (HSHF) diet group were fed a HSHF diet (20\% lard, 20\% sucrose, $2.5 \%$ cholesterol, $1 \%$ cholic acid salt, and $56.5 \%$ standard chow diet). These two groups simultaneously received saline by gavage. The mice in the HSHF $+\mathrm{S}$ group were maintained on the HSHF diet with Shujiangzhi (simvastatin, Merck \& Co., Inc, Hangzhou, China) administration by gavage at a dosage of $1 \mu \mathrm{g} / \mathrm{g} \cdot \mathrm{d}$ for 12 weeks. The other three groups were maintained on the HSHF diet with a gavage of krill oil (purchased from Sino-Ocean Co., Ltd., Dalian, China) by gavage at low (100 $\mathrm{g} / \mathrm{g} \cdot \mathrm{d}$, HSHF+LD group), moderate $(200 \mu \mathrm{g} / \mathrm{g} \cdot \mathrm{d}, \mathrm{HSHF}+\mathrm{MD}$ group), and high dosages $(600 \mu \mathrm{g} / \mathrm{g} \cdot \mathrm{d}, \mathrm{HSHF}+\mathrm{HD}$ group) for 12 weeks. The mice were 
kept in the same house during the experiments, and none of the mice died.

The body weights and food intake were measured weekly. After 12 weeks, fecal samples were collected again from each cage (representing four mice). Blood samples were collected from each mouse and immediately stored at $-80^{\circ} \mathrm{C}$. Organs from each mouse, including the brain, kidney, heart, liver, spleen, and lung, were excised and weighed. A viscera index was calculated using the formula: organ weight/body weight $(\mathrm{mg} / \mathrm{g})$.

\section{Measurement of Blood Biochemical Indices}

The TC, TG, HDL, and LDL levels in the blood samples were measured using kits from the Jiancheng Bioengineering Institute (Nanjing, China).

\section{Measurement of the Krill Oil Component}

The fatty acid composition was measured via chromatographymass spectrometry (GC-MS) as previously described (Satil et al., 2003). An Agilent 7890/M7-80EI system with a VOCOL column $(60 \mathrm{~m} \times 0.32 \mathrm{~mm})$ was used in this study, and helium was used as the carrier gas. The GC oven temperature began at $60^{\circ} \mathrm{C}$, increased to $260^{\circ} \mathrm{C}$ at a rate of $5^{\circ} \mathrm{C} / \mathrm{min}$, and then was maintained at $260^{\circ} \mathrm{C}$ for $40 \mathrm{~min}$. The gas flow rate was $50 \mathrm{~mL} / \mathrm{min}$. The temperature of the injector was maintained at $260^{\circ} \mathrm{C}$. The detected mass ranged from 30 to $425 \mathrm{~m} / \mathrm{z}$.

The astaxanthin concentration in the krill oil was measured via high-performance liquid chromatography (HPLC) as previously described (López-Cervantes et al., 2006). The HPLC was operated at a rate of $1 \mathrm{~mL} / \mathrm{min}$ with an SS Exil ODS column $(250 \times 4.6 \mathrm{~mm}, 5 \mu \mathrm{m})$ and detected at the $476 \mathrm{~nm}$ wavelength. A mixture of water, methanol, dichloromethane, and acetonitrile at a 4.5:28:22:45.5 ratio (v/v/v/v) was used as the mobile phase. An astaxanthin standard was purchased from Sigma-Aldrich Co. LLC (St. Louis, MO, USA).

\section{Total DNA Extraction, PCR, and Sequencing}

Total DNA was isolated from each sample (feces from the same cage) using a previously described method as Yu and Morrison (2004). The quantity of the extracted DNA was measured using a Thermo NanoDrop 2000C (Thermo Fisher Scientific, Waltham, MA, USA).

The PCR primers were designed based on the sequence of the V3 and V4 hypervariable regions of the bacterial 16S rRNA gene. The primers 319F 5'-ACTCCTACGGGAGGCAGC AG-3' and 806R 5'-GGACTACHVGGGTWTCTAAT-3' were designed with a barcode sequence that was unique to each sample. Amplification reactions were performed in $25 \mu \mathrm{L}$ volume containing $12.5 \mu \mathrm{L}$ Premix Ex TaqTM Hot Start Version (Takara Biotechnology Co. Ltd, Dalian, China), $0.1 \mu \mathrm{M}$ of each primer, and $20 \mathrm{ng}$ of template. Amplification was initiated at $98^{\circ} \mathrm{C}$ for $30 \mathrm{~s}$, followed by 35 cycles of denaturation at $98^{\circ} \mathrm{C}$ for $10 \mathrm{~s}$, primer annealing at $54^{\circ} \mathrm{C}$ for $30 \mathrm{~s}$, extension at $72^{\circ} \mathrm{C}$ for $45 \mathrm{~s}$, and final extension for $10 \mathrm{~min}$. PCR reactions for each sample were performed with a negative control in each run. The presence of amplicons was confirmed using gel electrophoresis, and the PCR products were normalized using AxyPrepTM Mag PCR Normalizer and then sequenced using the MiSeq system constructed with the Illumina Nextera XT Index kit in Sangon Biotech Co., Ltd. (Shanghai, China). Sequencing was performed on an Illumina MiSeq (Illumina, San Diego, CA) using $2 \times$ $300 \mathrm{bp}$ paired-end sequencing and multiple sequencing runs in accordance with the manufacturer's instructions.

\section{Data Analysis and Statistical Analysis}

Raw FASTQ files were multiplexed and filtered using QIIME (version 1.8.0) (Caporaso et al., 2010) according to the following steps: (1) the 300-bp reads were truncated at any position when an average quality score $<20$ on a 50 -bp sliding window was observed, and truncated reads shorter than 50-bp were removed; (2) data belonging to each sample were identified via the barcode sequence, and reads containing undetected nucleotides $(\mathrm{N})$ or two-nucleotide primer mismatches were removed; and (3) sequences that overlapped more than 10-bp were assembled, and the reads that could not be assembled were discarded. Operational taxonomic units (OTUs) were clustered using Usearch (version 7.1) (Edgar, 2010) at a similarity level of $97 \%$. The most abundant sequence of each OTU in all the samples was selected as the representative sequence. The RDP classifier (Wang et al., 2007) was used to annotate the representative OTU sequences, and taxonomic information was obtained for each OTU. The phylogenetic tree and the normalized relative abundance of OTUs were used for weighted and unweighted UniFrac principal coordinate analysis ( $\mathrm{PCoA}$ ) via vegan package in $\mathrm{R}$ software (version 3.2). The community richness index and community diversity were calculated using Mothur (version 1.30.1) and the reads number normalized to the same number (smallest) to reduce biases in sequencing depth for alpha and beta diversity analysis (Schloss et al., 2009).

All data are shown as the means \pm S.D. The ANOVA test and Tukey's post-hoc test (SPSS, version 19.0, Chicago, IL, USA) were used to analyse data with a normal distribution, and the Mann-Whitney test (MATLAB R2012a, Natick, MA, USA) was used to analyse data that did not meet the assumptions of the ANOVA. $P<0.05$ was defined as the standard criterion for statistical significance. Redundancy analysis models were constructed to identify specific bacterial phylotypes whose abundance was changed by HSHF feeding and by krill oil treatment. The relative abundance of each OTU was normalized and log-transformed, and used to construct RDA models to find the OTUs that were different among groups with Canoco for Windows 4.5 (Microcomputer Power, Ithaca, NY, USA) according to the manufacturer's instructions, and the type of treatment and dose of krill oil were used as environmental variables. Responding OTUs that explained more than $4 \%$ of the variability of the samples selected. Statistical significance was assessed by MCPP with 499 random permutations under the full model. Correlations between gut microbial composition (OTU abundances) and the average of the host parameters of hyperlipidemia in the same cage were identified using Spearman's correlation (SPSS, version 19.0, Chicago, IL, USA). Correlations between gut microbial composition and the average of the host parameters in the same cage were considered significant when 
$P<0.05$. False discovery rate control was used to account for multiple comparisons when evaluating correlations between OTUs and host parameters in SPSS software, and correlations were deemed significant at false discovery rate $<0.25$.

\section{Accession Number}

The sequences have been deposited in the NCBI Sequence Read Archive Database under accession number SRP095937.

\section{RESULTS}

\section{The Major Components of Krill Oil}

The krill oil purchased from Sino-Ocean Co., Ltd. (Dalian, China; shown in Figure S1) was a transparent, dark red, and viscous oil. The krill oil contained $30.04 \mathrm{wt} \%$ unsaturated fatty acids. The DHA and EPA concentrations were 2.62 wt \% and 5.56 wt \%, respectively. Eighty percent of the total fatty acids in the krill oil were phospholipids (568 mg/g; Table S1). The astaxanthin concentration was $37.43 \mathrm{mg} / 100 \mathrm{~g}$.

\section{Krill Oil Attenuates Features of Hyperlipidemia in HSHF Diet Fed Mice}

The mice in the HSHF group gained more weight than the mice in the control group $(P<0.001$; Figure 1A, Table S2). The mice in the HSHF group developed the hallmark features of hyperlipidemia, with a significant increase in the LDL, TC, and TG concentrations $(P<0.001)$ and a significant decrease in the HDL concentration $(P<0.01$; Figure 2$)$. Additionally, the mice in the HSHF group experienced a significant increase in their liver weights, the liver index increased by $19.4 \%(P<0.001)$, and the other visceral indices remained unchanged (Figure 1B, Figure S2).

Twelve weeks of dietary supplementation with different doses of krill oil significantly attenuated the HSHF diet-induced weight gain (Figure 1A, Table S2), although no differences were observed in the food intake (Figure 1C). Furthermore, krill oil treatment significantly attenuated hyperlipidemia in the mice fed the HSHF diet. The LDL, TC, and TG levels were significantly decreased in the HSHF+MD group $(P<0.05)$ and the HSHF+HD group $(P<0.01)$ compared to those in the HSHF group. The HSHF+LD group showed a significant reduction in the TC level $(P<0.05$; Figure 2, Table S3). Krill oil supplementation decreased the liver index of all groups compared to that of the HSHF group, with reduction of 5.6, 5.3, and $6.6 \%(P>0.05)$ in the HSHF+LD, HSHF+MD, and HSHF+HD groups, respectively. The other visceral indices showed no response to krill oil supplementation at any of the doses (Figure 1B, Figure S2). The HSHF $+\mathrm{S}$ group showed the same pattern for the biochemical indices and liver index as the HSHF+HD group. Altogether, these results demonstrated the dose-dependent effect and safety of krill oil in mitigating HSHF diet induced weight gain and hyperlipidemia symptoms.

\section{The HSHF Diet and Krill Oil Treatment Lead to Changes in the Gut Microbiota Structure}

To elucidate the contributions of the krill oil treatment to the gut microbiota, we collected fecal samples from 72 mice after 12 weeks of treatment and performed a high-throughput sequencing analysis of the V3 and V4 hypervariable regions of the bacterial $16 \mathrm{~S}$ rRNA gene. And the raw reads in each group are: control group $(39,207,36,383$, and 26,214), HSHF group (40,795, 31,757, and 21,003$)$, HSHF+LD group $(21,404,41,845$, and 23,910$)$, HSHF+MD group $(37,709,45,256$, and 30,429), HSHF+HD group $(27,117,29,835$, and 26,325$)$, and $\mathrm{HSHF}+\mathrm{S}$ group $(34,849$, $30,748$, and 19,576$)$.

The diversity of the microbial communities was measured using the Shannon and Simpson diversity indices (Figure S3). A significantly decreased Shannon index $(P<0.05)$ and a significantly increased Simpson index $(P<0.05)$ were measured in the HSHF group compared with those in the control group, which indicated that bacterial diversity was decreased after the HSHF diet treatment. The krill oil treatment restored the bacterial diversity regardless of the krill oil dose, with an increased Shannon index as well as a reduced Simpson index compared with those in the HSHF group.
A

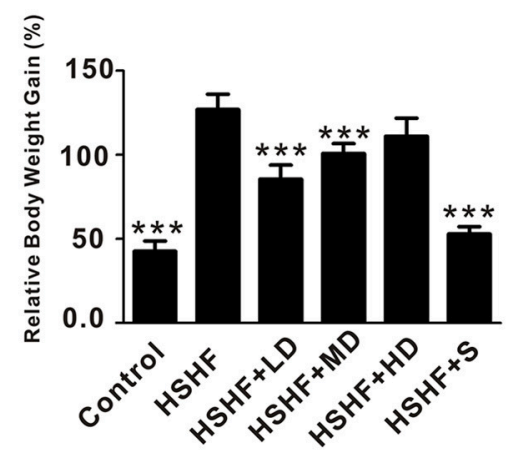

B

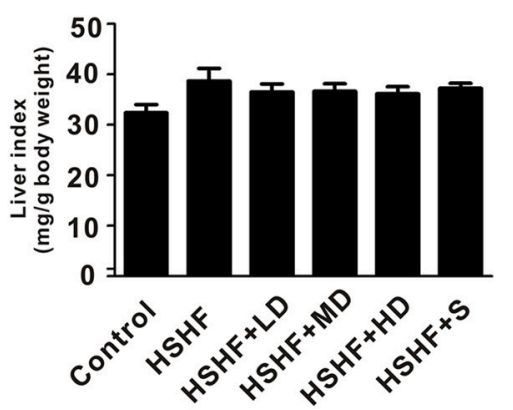

C

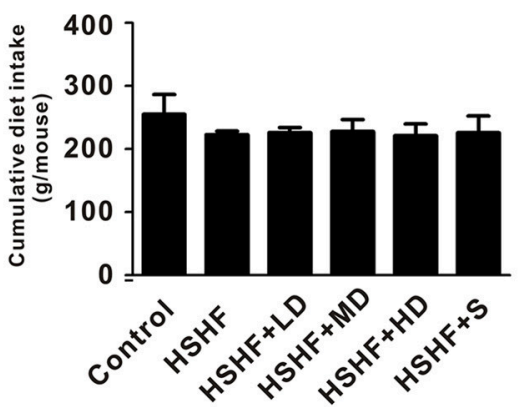

FIGURE 1 | Effects of krill oil treatment on the physical features of mice fed the HSHF diet. (A) The relative body weight gain of HSHF diet fed mice with krill oil supplementation. (B) Liver index of HSHF diet fed mice with krill oil supplementation. (C) Total food intake of HSHF diet fed mice with krill oil supplementation. TC, total cholesterol. TG, triglyceride. HDL, high-density lipoprotein. LDL, low-density lipoprotein. Data are presented as the means \pm S.D. Differences were assessed by ANOVA. ${ }^{* \star} P<0.001$ vs. HSHF group. 


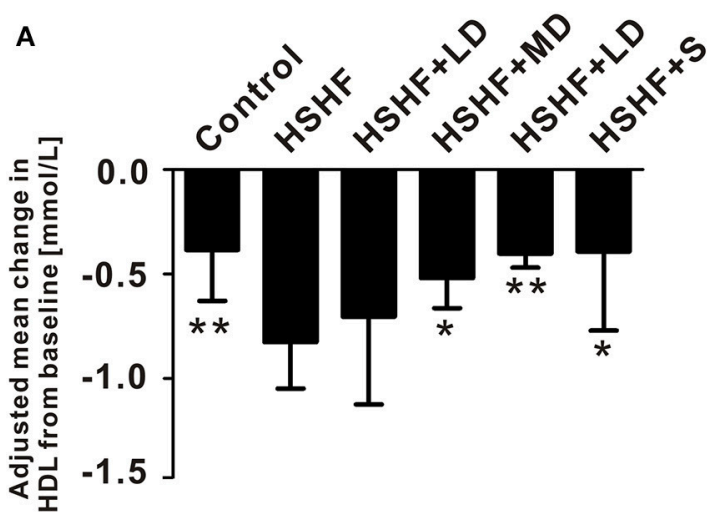

B

C

D
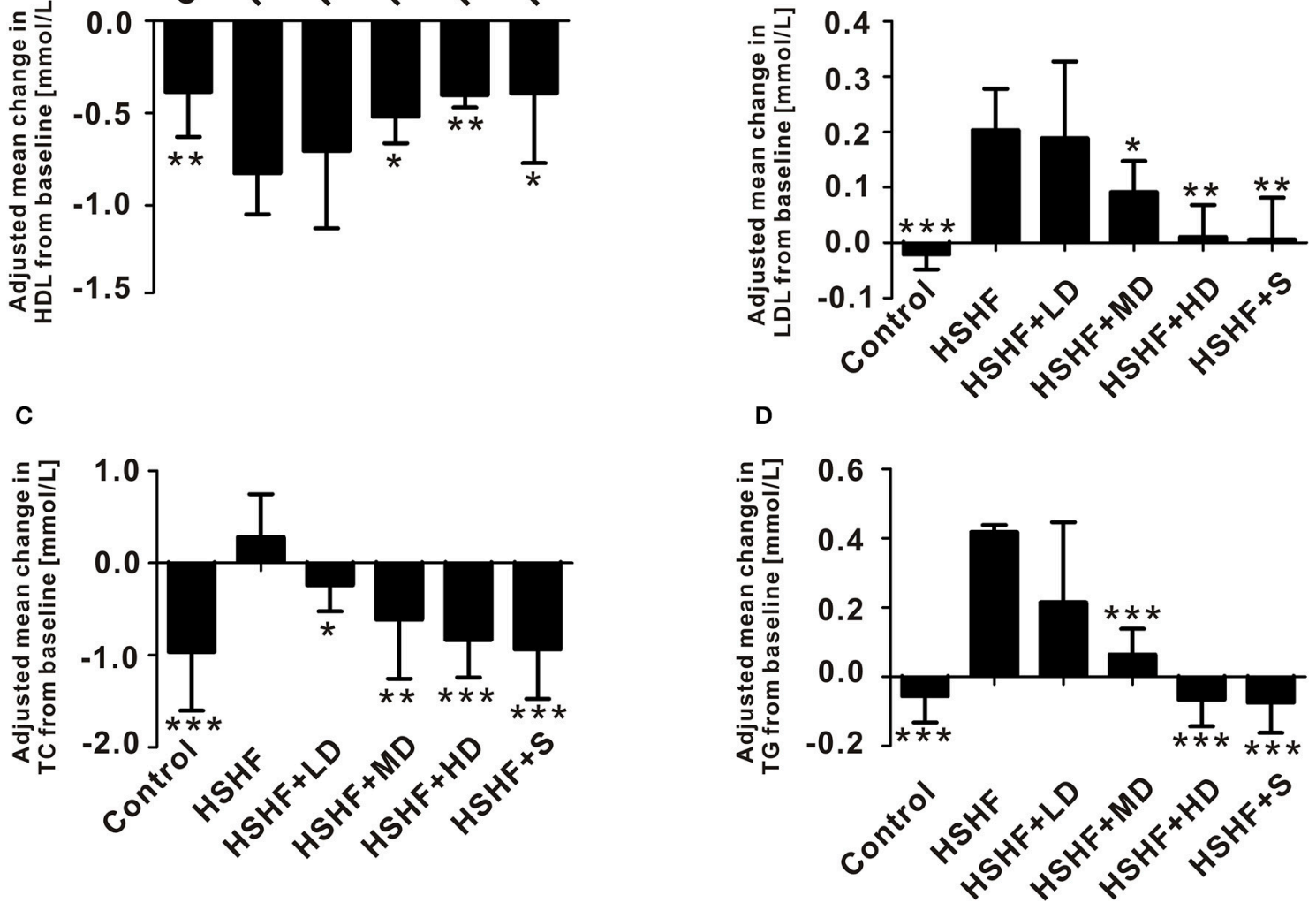

FIGURE 2 | Krill oil significantly improved serum lipid control in mice fed the HSHF diet. (A) Change in the HDL level. (B) Change in the LDL level. (C) Change in the TC level. (D) Change in the TG level. Data are presented as the means \pm S.D. Differences were assessed by ANOVA. ${ }^{\star} P<0.05,{ }^{\star \star} P<0.01$, and ${ }^{\star \star \star} P<0.001$ vs. HSHF group.

The overall compositions of the gut microbiota in the six groups were analyzed via Weighted and Unweighted UniFrac PCoA (Figure 3, Figure S4). The low- and moderate-dose krill oil treatments shifted the overall compositions of the gut microbiota in the HSHF group toward the composition of the control group, whereas the HSHF+HD group showed a unique pattern that was different from that in the HSHF and control groups. Interestingly, we identified a close relationship between the gut microbiota structures of the HSHF and HSHF+S, even though the LDL, TC, and TG indices were significantly reduced and the hyperlipidemia features were attenuated in the HSHF+S group. This finding indicated that the anti-hyperlipidemic activity of simvastatin was not caused by modulation of the gut microbiota.

\section{Microbial Shifts in Response to the Krill Oil Treatment in Mice Fed the HSHF Diet}

The RDP classifier clustered $98.93 \%$ of the reads into 15 phyla. The most abundant phyla in the control group were: Proteobacteria, Firmicutes, Cyanobacteria, Bacteroidetes, Actinobacteria, and Thaumarchaeota. After 12 weeks on HSHF

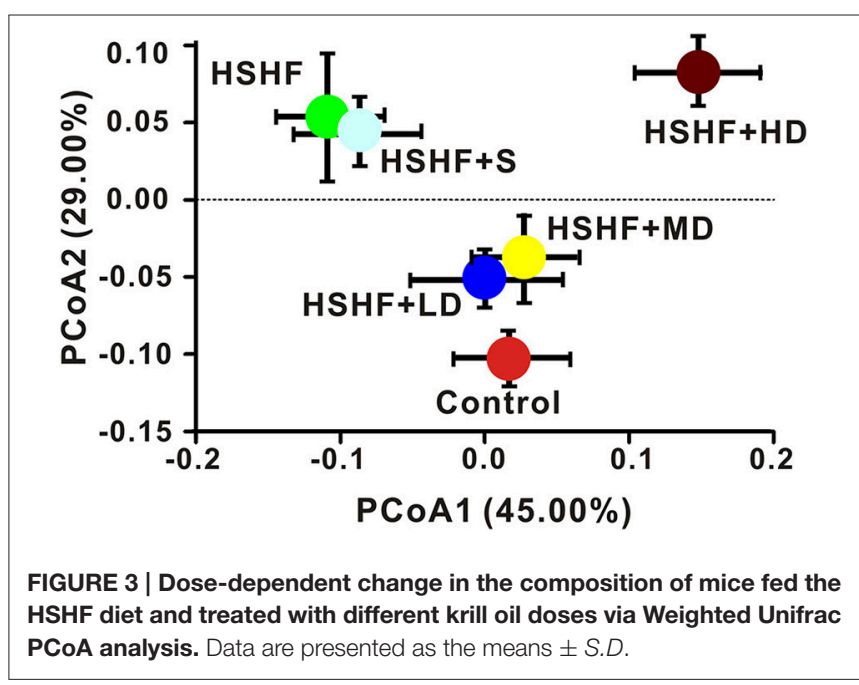

diet, widespread changes in the structure of the gut microbiota were observed at the phylum level compared with that in the control group, with significant decreased proportions of 
Cyanobacteria $(P<0.05)$, Thaumarchaeota $(P<0.05)$, and significant increased proportions of Firmicutes $(P<0.05)$. The low and moderate-dose krill oil treatment attenuated the changes in the gut microbiota structures, whereas the high-dose treatment aggravated the changes in some phyla (Figure 4A, Table S4). However, the ratio of Firmicutes to Bacteroidetes increased after HSHF diet treatment $(P<0.01)$, whereas the krill oil treatment attenuated this increase $(P<0.01)$.

Bacilli, Erysipelotrichia, and Clostridia are common classes in phylum Firmicutes. The abundance of Erysipelotrichia significantly increased $(P<0.05)$ after the HSHF diet treatment, whereas the abundance of Bacilli and Clostridia decreased, although not reaching significant level. The extra addition of krill oil restored the abundance of these three classes regardless of the krill oil dose (Figure 4B, Table S5). The classes Gammaproteobacteria, Betaproteobacteria, and Alphaproteobacteria were the major classes in the Proteobacteria phylum. The abundances of these three classes increased in the HSHF+LD and HSHF+MD groups. However, the opposite change was observed in the $\mathrm{HSHF}+\mathrm{HD}$ and $\mathrm{HSHF}+\mathrm{S}$ groups (Figure 4B, Table S5).

A total of $385,189,311,382,163$, and 173 genera were identified from the control, HSHF, HSHF+LD, HSHF+MD,
$\mathrm{HSHF}+\mathrm{HD}$, and $\mathrm{HSHF}+\mathrm{S}$ groups, respectively. Genera with $>1 \%$ abundance in at least four groups were defined as abundant genera. Seven abundant genera were identified (Lactobacillus, Bacillus, Streptococcus, Staphylococcus, Delftia, Ralstonia, and Serratia; Table S6). The first four genera belong to phylum Firmicutes, and the latter three belong to phylum Proteobacteria. Among the seven abundant genera, only the detected abundance of Ralstonia increased after the HSHF diet treatment $(P>0.05)$, whereas the species in the genera Streptococcus and Delftia were significantly decreased (Table S6). Furthermore, the different doses of krill oil had various effects on the abundances of these seven genera. Detailed information is provided in Table $\mathbf{S 6 .}$

\section{Key Phylotypes Respond to Krill Oil Treatment in Mice Fed with HSHF Diet}

A total of 2,173,1,352, 1,860,2,064, 1,226, and 1,259 OTUs were identified in the control, HSHF, HSHF+LD, HSHF+MD, $\mathrm{HSHF}+\mathrm{HD}$, and $\mathrm{HSHF}+\mathrm{S}$ groups, respectively. Because the antihyperlipidemic activity of simvastatin did not occur through modulation of the gut microbiota, further analysis was performed in these groups, except for the HSHF+S group. Sixty-five key OTUs that responded to the krill oil treatment were identified

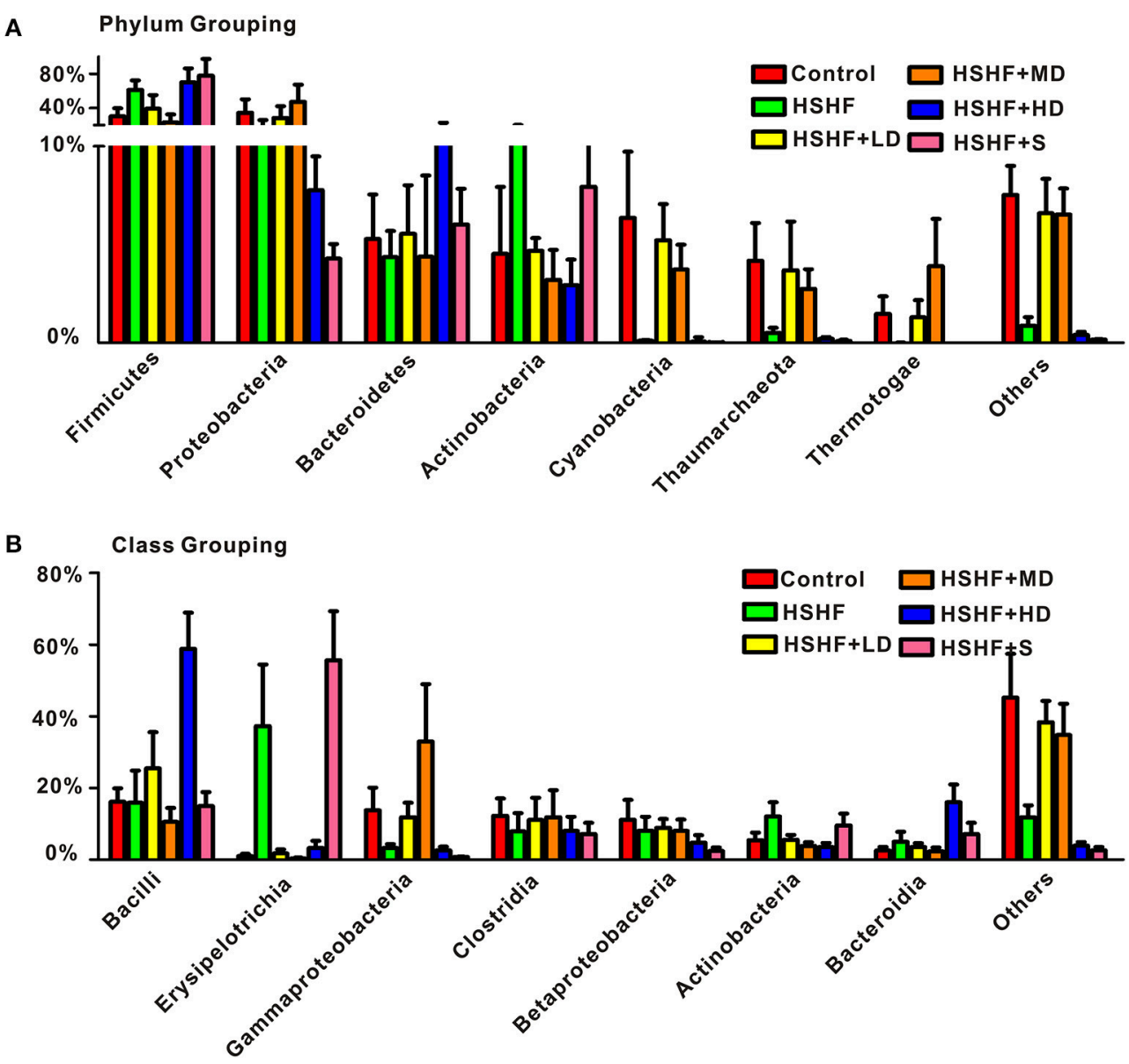

FIGURE 4 | RDP classifications of the average sequences reads. (A) At the phylum level and (B) at the class level. Data are presented as the means \pm S.D. 
via redundancy analysis (Figure 5, Tables S7, S8). Among the 65 key OTUs, 26 OTUs were decreased by the HSHF diet and then increased by the krill oil treatment regardless of the dose, whereas the opposite pattern occurred for 39 OTUs. Among the 26 OTUs increased by krill oil, only OTU3840, which belongs to the Haemophilus genus, showed a significant negative correlation with the TC $(R=-0.92, P<0.01)$, TG $(R=-0.88, P<0.05)$ and LDL levels $(R=-0.94$, $P<0.01)$ and a significant positive correlation with the HDL level $(R=0.90, P<0.01)$. Four OTUs (OTU80, OTU6976, OTU1,

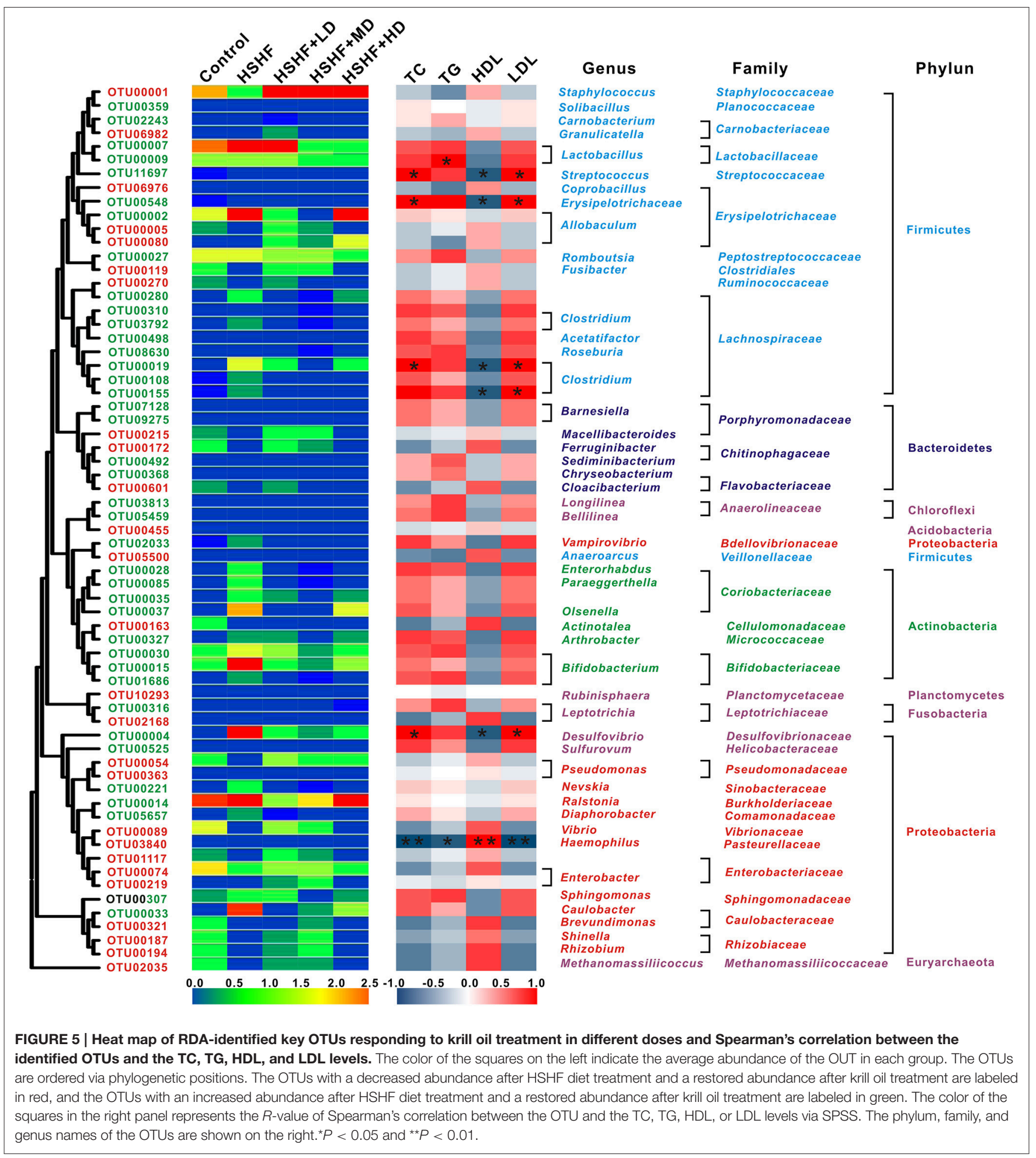


and OTU3840) from Allobaculum, Coprobacillus, Staphylococcus, and Haemophilus, respectively, showed the highest relative abundance after the high-dose krill oil (Figure 5, Tables S7, S8). Among the 39 OTUs that were decreased by krill oil treatment, 5 OTUs showed a significant positive correlation with the TC $(R=0.91, P<0.05)$ and LDL $(R=0.90, P$ $<0.05)$ and a significant negative correlation with the HDL concentration $(R=0.93, P<0.05)$. These OTUs belonged to Erysipelotrichaceae $(n=1)$, Clostridium XlVa $(n=2)$, Streptococcus $(n=1)$, and Desulfovibrio $(n=1)$. OTU9, which belongs to Lactobacillus, had a significant positive correlation only with the TG concentration $(R=0.92, P<0.05)$. Seven OTUs (OTU492, OTU5459, OTU3813, OTU548, OTU9, OTU27, and OTU316) from Sediminibacterium, Bellilinea, Longilinea, Erysipelotrichaceae, Lactobacillus, Romboutsia, and Leptotrichia, respectively, showed the lowest relative abundance after HD krill oil treatment (Figure 5, Tables S7, S8).

\section{DISCUSSION}

To the best of our knowledge, this study is the first to elucidate the dose-dependent effect of krill oil on hyperlipidemia in a mouse model via a randomized design. In this study, the krill oil treatment provided significant and meaningful dosedependent reductions in the physical (relative body weight gain and liver index; Figure 1, Table S2) and biochemical indices (LDL, TC, and TG; Figure 2) compared with those of the HSHF group. Additionally, the HDL level was significantly improved after krill oil treatment regardless of the dose. These findings were consistent with previous studies on dietary krill oil supplementation in mice fed a high-fat diet and in overweight humans (Maki et al., 2009; Tandy et al., 2009; Burri et al., 2011).

Krill oil supplementary in diet with low and moderate doses in this study maintained the structure of the gut microbiota like the control group, as well as the levels of HDL, LDL, TC, and TG. Previous studies have indicated that diet has a great influence (estimated at $57 \%$, compared with $12 \%$ for genetic factors) on the structure of the gut microbiota (Tomasello et al., 2011). Consumption of a Western diet results in an increased abundance of Bacteroides spp, Ruminococcus torques, and Bilophila wadsworthia (Kamada et al., 2013), whereas the microbiota population with an Eastern diet has a higher abundance of Prevotella spp. (Ananthakrishnan, 2015). The improvement of vascular dysfunction by inulin-type fructans supplementary in the diet is linked to the increased nitric oxide producing bacteria, replenishment of abundance in Akkermansia and decreased abundance in bacterial taxa involved in secondary bile acid synthesis (Catry et al., 2017). The high consumption of fiber in the diet modified the gut microbiota populations and increased the abundance of acetate-producing bacteria (Marques et al., 2017).

However, the high-dose group, which exhibited further alleviation of the hyperlipidemia symptoms, showed a unique gut microbiota structure compared to that of the HSHF and control groups. Compared with the LD and MD groups, the
HD group showed a decreased gut microbial diversity and the Proteobacteria abundance, and an increase in the Firmicutes abundance, although the Firmicutes to Bacteroidetes ratio was almost the same as the ratio in the MD group. The change in the gut microbiota structure in the HD group was similar to the previously "obesity model" (Marchesi et al., 2015; Alard et al., 2016; Denou et al., 2016). Krill oil and fish oil are mixtures of saturated and unsaturated fatty acids. In this study, a high concentration of supplementary fatty acids led to an increased body weight gain from $18.58 \mathrm{~g}$ in the LD group to $23.58 \mathrm{~g}$ in the HD group (Figure 1A). These results indicated that the krill oil treatment will lead to two effects in the same time: (1) the obesity and (2) the alleviation of hyperlipidemia. When krill oil intake at a low concentration $(100$ or $200 \mu \mathrm{g} / \mathrm{g} \cdot \mathrm{d})$, the main effect focused on hyperlipidemia alleviation rather than obesity, and a control-like gut microbiota structure were observed in this study. When the concentration of krill oil increased to $600 \mu \mathrm{g} / \mathrm{g} \cdot \mathrm{d}$, the effect on obesity was detected on increased body weight gain compared with the LD and MD groups, as well as the obesity-like structure with increased Firmicutes and decreased gut microbial diversity.

Seven abundant genera were detected in this study (Table S6), and the change in the abundance of these genera might explain the anti-hyperlipidemic effect. Lactobacillus and Streptococcus, which are lactic acid bacteria, were enriched by krill oil treatment in our study. The oral administration of Lactobacillus and Streptococcus successfully influenced the TC, TG, LDL, and HDL levels in addition to aminotransferase and aspartate aminotransferase activities in the rats with experimentally induced hyperlipidemia (Aderiye et al., 2007; Ngongang et al., 2016). The increased proportion of Serratia after krill oil treatment led to the accumulation of the compound FR177391, which had an anti-hyperlipidemic effect (Sato et al., 2005). In addition to hyperlipidemia, krill oil supplementation was also beneficial for the treatment of inflammatory and neurological disorders, and inflammatory disease was positively correlated with the Ralstonia and Delftia abundance (Tyler et al., 2013; Chen et al., 2014c; Bilgin et al., 2015; Tamburini and Clemente, 2016). In this study, krill oil treatment significantly decreased the proportion of Ralstonia, which might explain the anti-inflammatory effect. However, the reason for the unexpected increased abundance of Delftia remains unknown.

Among the 65 key OTUs that responded to krill oil treatment, 26 OTUs were increased and 39 were decreased, compared with those of the HSHF group, regardless of the krill oil dose (Figure 5). The OTUs that were increased in abundance after krill oil treatment, including the genera Allobaculum and Enterobacter, which were negatively correlated with hyperlipidemia phenotypes, and thus might represent potentially beneficial bacteria. The OTUs that showed reduced proportions, including the genera Barnesiella, Clostridium XlVa, Desulfovibrionaceae, and Lactobacillus, might include some harmful bacteria. Additionally, krill oil treatment led to the reduced abundance of Desulfovibrionaceae and Clostridium $\mathrm{XIVa}$ and increased proportion of Allobaculum. Allobaculum was shown to prevent dextran sulfate sodium-induced 
inflammation, whereas Desulfovibrionaceae and Clostridium $\mathrm{XIVa}$ that were involved in the obesity-related metabolic disorders or pro-inflammatory reactions (Zhang et al., 2010, 2012; Lam et al., 2012; Le Roy et al., 2013; Tuovinen et al., 2013).

However, among the OTUs detected both in the control and HSHF groups, 11 and 8 OTUs increased whereas 6 and 8 OTUs decreased after HSHF treatment, which belonged to Bifidobacterium probiotics and Lactobacillus, respectively. This finding indicated that many OTUs belonging to the same genus correlated in opposite directions. Previous studies have shown that different bacterial species in the same genus may respond differentially to the same environmental stressor, such as changing from a high-fat to a standard diet (Zhang et al., 2010, 2012). For instance, both Bifidobacterium animalis ssp. lactis GCL2505 and B. longum ssp. longum JCM1217T belong to genus Bifidobacterium but show different effects on the reduction of visceral fat accumulation, glucose tolerance improvement, and gut microbiota modulation (Aoki et al., 2017). Thus, identifying species-level phylotypes changes in response to treatment is important.

In conclusion, the results obtained in this study suggest that structural alterations in the gut microbiota induced by krill oil treatment are dose-dependent and associated with the alleviation of hyperlipidemia. Krill oil treatment will lead to the obesity and the alleviation of hyperlipidemia in the same time. The effects on the obesity was not obvious after low and moderate dose krill oil treatment, and a control-like gut microbiota structure with increased abundance of Serratia, Delftia, and Streptococcus in the gut and decreased proportion of Ralstonia were observed. Whereas with the increase of krill oil concentration, the effect on obesity was detected on increased body weight gain compared with the LD and MD groups, as well as the obesity-like gut microbiota structure with increased Firmicutes and decreased gut microbial diversity.

\section{AUTHOR CONTRIBUTIONS}

CL and XS designed the study. CL, TS, YL, DZ, and JZ performed the experiments. CL, TS, YL, DZ, JZ, and XS provided reagents and materials. CL and TS analyzed the data. CL, TS, and XS wrote the main manuscript text and prepared the figures. All authors reviewed the manuscript.

\section{REFERENCES}

Abliz, A., Aji, Q., Abdusalam, E., Sun, X., Abdurahman, A., Zhou, W., et al. (2014). Effect of Cydonia oblonga Mill. leaf extract on serum lipids and liver function in a rat model of hyperlipidaemia. J. Ethnopharmacol. 151, 970-974. doi: 10.1016/j.jep.2013.12.010

Aderiye, B., Laleye, S., and Odeyemi, A. (2007). Hypolipidemic potential of Lactobacillus and Streptococcus spp. from some Nigerian fermented foods. Res. J. Microbiol. 2, 538-544. doi: 10.3923/jm.2007.538.544

Alard, J., Lehrter, V., Rhimi, M., Mangin, I., Peucelle, V., Abraham, A. L., et al. (2016). Beneficial metabolic effects of selected probiotics on diet-induced obesity and insulin resistance in mice are associated with

\section{FUNDING}

This work was supported by the Regional Demonstration Project of Marine Economic Innovation and Development (2013710), K. C. Wong Magna Fund in Ningbo University.

\section{ACKNOWLEDGMENTS}

We thank Nature Research Editing Service for English language editing.

\section{SUPPLEMENTARY MATERIAL}

The Supplementary Material for this article can be found online at: http://journal.frontiersin.org/article/10.3389/fmicb. 2017.00905/full\#supplementary-material

Table S1 | The fatty acid components of the krill oil.

Table S2 | The body weight of mice fed experimental diets. Data are presented as the means \pm S.D.

Table S3 | Serum lipid indices of mice fed experimental diets. Data are presented as the means $\pm S$.D. Differences were assessed by ANOVA. ${ }^{*} P<$ 0.05 , ${ }^{* *} P<0.01,{ }^{* * *} P<0.001$, compared with the HSHF group.

Table S4 | The RDP classifications of the sequence reads at the phylum level. Data are presented as the means $\pm S$.D. Differences were assessed by ANOVA. ${ }^{*} P<0.05$, compared with the HSHF group.

Table S5 | The RDP classifications of the sequence reads at the class level. Data are presented as the means $\pm S$.D. Differences were assessed by ANOVA. ${ }^{*} P<0.05$, compared with the HSHF group.

Table S6 | Seven abundant genera in all groups. Data are presented as the means \pm S.D. $* P<0.05$, compared with the HSHF group.

Table S7 | Sixty-five key OTUs respond to krill oil treatment as identified by redundancy analysis (RDA).

Table S8 | Relative average abundance of the 65 key OTUs responding to krill oil treatment as identified by redundancy analysis (RDA).

Figure S1 | Features of the krill oil. (A) Concentrated krill oil. (B) Diluted krill oil.

Figure S2 | Viscera indices of the mice fed the HSHF diet with krill oil supplementation. Data are presented as the means \pm S.D.

Figure S3 | Different microbial diversity indices in different groups. (A) ACE index. (B) Chao1 index. (C) Shannon index. (D) Simpson. ${ }^{*} P<0.05$ vs. control group.

Figure S4 | Dose-dependent change in the composition of mice fed the HSHF diet and treated with different krill oil doses via unweighted Unifrac PCoA analysis. Data are presented as the means \pm S.D.

improvement of dysbiotic gut microbiota. Environ. Microbiol. 111, 1484-1497. doi: 10.1111/1462-2920.13181

Ananthakrishnan, A. N. (2015). Epidemiology and risk factors for IBD. Nat. Rev. Gastroenterol. Hepatol. 12:205. doi: 10.1038/nrgastro.2015.34

Aoki, R., Kamikado, K., Suda, W., Takii, H., Mikami, Y., Suganuma, N., et al. (2017). A proliferative probiotic Bifidobacterium strain in the gut ameliorates progression of metabolic disorders via microbiota modulation and acetate elevation. Sci. Rep. 7:43522. doi: 10.1038/srep 43522

Araujo, P., Zhu, H., Breivik, J. F., Hjelle, J. I., and Zeng, Y. (2014). Determination and structural elucidation of triacylglycerols in krill oil by chromatographic techniques. Lipids 49, 163-172. doi: 10.1007/s11745-013-3855-6 
Axling, U., Olsson, C., Xu, J., Fernandez, C., Larsson, S., Ström, K., et al. (2012). Green tea powder and Lactobacillus plantarum affect gut microbiota, lipid metabolism and inflammation in high-fat fed C57BL/6J mice. Nutr. Metab. 9:105. doi: 10.1186/1743-7075-9-105

Bilgin, H., Sarmis, A., Tigen, E., Soyletir, G., and Mulazimoglu, L. (2015). Delftia acidovorans: a rare pathogen in immunocompetent and immunocompromised patients. Can. J. Infect. Dis. Med. Microbiol. 26, 277-279. doi: 10.1155/2015/973284

Bunea, R., El Farrah, K., and Deutsch, L. (2004). Evaluation of the effects of Neptune Krill Oil on the clinical course of hyperlipidemia. Altern. Med. Rev. 9, 420-428.

Burri, L., Berge, K., Wibrand, K., Berge, R. K., and Barger, J. L. (2011). Differential effects of krill oil and fish oil on the hepatic transcriptome in mice. Front. Genet. 2:45. doi: 10.3389/fgene.2011.00045

Caporaso, J. G., Kuczynski, J., Stombaugh, J., Bittinger, K., Bushman, F. D., Costello, E. K., et al. (2010). QIIME allows analysis of high-throughput community sequencing data. Nat. Methods 7:335. doi: 10.1038/nmeth. f.303

Catry, E., Bindels, L. B., Tailleux, A., Lestavel, S., Neyrinck, A. M., Goossens, J.-F., et al. (2017). Targeting the gut microbiota with inulin-type fructans: preclinical demonstration of a novel approach in the management of endothelial dysfunction. Gut. doi: 10.1136/gutjnl-2016-313316. [Epub ahead of print].

Chen, D., Yang, Z., Chen, X., Huang, Y., Yin, B., Guo, F., et al. (2014a). The effect of Lactobacillus rhamnosus hsryfm 1301 on the intestinal microbiota of a hyperlipidemic rat model. BMC Complement. Altern. Med. 14:386. doi: 10.1186/1472-6882-14-386

Chen, H., Miao, H., Feng, Y.-L., Zhao, Y.-Y., and Lin, R.-C. (2014b). Metabolomics in dyslipidemia. Adv. Clin. Chem. 66, 101-119. doi: 10.1016/B978-0-12-801401-1.00004-9

Chen, L., Wang, W., Zhou, R., Ng, S. C., Li, J., Huang, M., et al. (2014c). Characteristics of fecal and mucosa-associated microbiota in Chinese patients with inflammatory bowel disease. Medicine 93:e51. doi: 10.1097/MD.0000000000000051

Denou, E., Marcinko, K., Surette, M. G., Steinberg, G. R., and Schertzer, J. D. (2016). High intensity exercise training increases the diversity and metabolic capacity of the mouse distal gut microbiota during diet-induced obesity. Am. J. Physiol. Endocrinol. Metab. 310:E982. doi: 10.1152/ajpendo.00537.2015

Deutsch, L. (2007). Evaluation of the effect of Neptune Krill Oil on chronic inflammation and arthritic symptoms. J. Am. Coll. Nutr. 26, 39-48. doi: 10.1080/07315724.2007.10719584

Eckel, R. H., Grundy, S. M., and Zimmet, P. Z. (2005). The metabolic syndrome. Lancet 365, 1415-1428. doi: 10.1016/S0140-6736(05)66378-7

Edgar, R. C. (2010). Search and clustering orders of magnitude faster than BLAST. Bioinformatics 26:2460. doi: 10.1093/bioinformatics/btq461

Everard, A., Lazarevic, V., Derrien, M., Girard, M., Muccioli, G. G., Neyrinck, A. M., et al. (2011). Responses of Gut microbiota and glucose and lipid metabolism to prebiotics in genetic obese and diet-induced leptin-resistant mice. Diabetes 60, 2775-2786. doi: 10.2337/db11-0227

Falcinelli, S., Picchietti, S., Rodiles, A., Cossignani, L., Merrifield, D. L., Taddei, A. R., et al. (2015). Lactobacillus rhamnosus lowers zebrafish lipid content by changing gut microbiota and host transcription of genes involved in lipid metabolism. Sci. Rep. 5:9336. doi: 10.1038/srep09336

Farnier, M., and Davignon, J. (1998). Current and future treatment of hyperlipidemia: the role of statins. Am. J. Cardiol. 82, 3J-10J. doi: 10.1016/S0002-9149(98)00423-8

Ferramosca, A., Conte, A., Burri, L., Berge, K., De Nuccio, F., Giudetti, A. M., et al. (2012). A krill oil supplemented diet suppresses hepatic steatosis in high-fat fed rats. PLoS ONE 7:e38797. doi: 10.1371/journal.pone.0038797

Grundy, S. M., Ahrens, E., Salen, G., Schreibman, P. H., and Nestel, P. J. (1972). Mechanisms of action of clofibrate on cholesterol metabolism in patients with hyperlipidemia. J. Lipid Res. 13, 531-551.

Harris, W., Zucker, M., and Dujovne, C. (1988). Omega-3 fatty acids in hypertriglyceridemic patients: triglycerides vs methyl esters. Am. J. Clin. Nutr. 48, 992-997.

He, K., Hu, Y., Ma, H., Zou, Z., Xiao, Y., Yang, Y., et al. (2016). Rhizoma Coptidis alkaloids alleviate hyperlipidemia in B6 mice by modulating gut microbiota and bile acid pathways. Biochim. Biophys. Acta Mol. Basis Dis. 1862, 1696-1709. doi: 10.1016/j.bbadis.2016.06.006
Hussein, G., Nakagawa, T., Goto, H., Shimada, Y., Matsumoto, K., Sankawa U., et al. (2007). Astaxanthin ameliorates features of metabolic syndrome in SHR/NDmcr-cp. Life Sci. 80, 522-529. doi: 10.1016/j.lfs.2006.09.041

Ierna, M., Kerr, A., Scales, H., Berge, K., and Griinari, M. (2010). Supplementation of diet with krill oil protects against experimental rheumatoid arthritis. BMC Musculoskelet. Disord. 11:136. doi: 10.1186/1471-2474-11-136

Ikeuchi, M., Koyama, T., Takahashi, J., and Yazawa, K. (2007). Effects of astaxanthin in obese mice fed a high-fat diet. Biosci. Biotechnol. Biochem. 71, 893-899. doi: 10.1271/bbb.60521

Kamada, N., Seo, S. U., Chen, G. Y., and Nú-ez, G. (2013). Role of the gut microbiota in immunity and inflammatory disease. Nat. Rev. Immunol. 13, 321-335. doi: 10.1038/nri3430

Kim, C. H., Mitchell, J. B., Bursill, C. A., Sowers, A. L., Thetford, A., Cook, J. A., et al. (2015). The nitroxide radical TEMPOL prevents obesity, hyperlipidaemia, elevation of inflammatory cytokines, and modulates atherosclerotic plaque composition in apoE-/- mice. Atherosclerosis 240, 234-241. doi: 10.1016/j.atherosclerosis.2015.03.012

Kong, W., Wei, J., Abidi, P., Lin, M., Inaba, S., Li, C., et al. (2004). Berberine is a novel cholesterol-lowering drug working through a unique mechanism distinct from stains. Nat. Med. 10, 1344-1351. doi: 10.1038/nm1135

Lam, Y. Y., Ha, C. W., Campbell, C. R., Mitchell, A. J., Dinudom, A., Oscarsson, J., et al. (2012). Increased gut permeability and microbiota change associate with mesenteric fat inflammation and metabolic dysfunction in diet-induced obese mice. PLoS ONE 7:e34233. doi: 10.1371/journal.pone.0034233

Le Roy, T., Llopis, M., Lepage, P., Bruneau, A., Rabot, S., Bevilacqua, C. et al. (2013). Intestinal microbiota determines development of non-alcoholic fatty liver disease in mice. Gut 62, 1787-1794. doi: 10.1136/gutjnl-2012303816

Ley, R. E., Turnbaugh, P. J., Klein, S., and Gordon, J. I. (2006). Microbial ecology: human gut microbes associated with obesity. Nature 444, 1022-1023. doi: $10.1038 / 4441022$ a

López-Cervantes, J., Sánchez-Machado, D. I., Gutiérrez-Coronado, M. A., and Ríos-Vázquez, N. J. (2006). Quantification of astaxanthin in shrimp waste hydrolysate by HPLC. Biomed. Chromatogr. 20, 981-984. doi: 10.1002/bmc.676

Maki, K. C., Reeves, M. S., Farmer, M., Griinari, M., Berge, K., Vik, H., et al. (2009). Krill oil supplementation increases plasma concentrations of eicosapentaenoic and docosahexaenoic acids in overweight and obese men and women. Nutr. Res. 29, 609-615. doi: 10.1016/j.nutres.2009.09.004

Marchesi, J. R., Adams, D. H., Fava, F., Hermes, G. D. A., Hirschfield, G. M., Hold, G., et al. (2015). The gut microbiota and host health: a new clinical frontier. Gut 65:330. doi: 10.1136/gutjnl-2015-309990

Marques, F. Z., Nelson, E., Chu, P.-Y., Horlock, D., Fiedler, A., Ziemann, M., et al. (2017). High fibre diet and acetate supplementation change the gut microbiota and prevent the development of hypertension and heart failure in hypertensive mice. Circulation 135, 964-977. doi: 10.1161/CIRCULATIONAHA.116. 024545

Ngongang, E. F. T., Tiencheu, B., Achidi, A. U., Fossi, B. T., Shiynyuy, D. M., Womeni, H. M., et al. (2016). Effects of probiotic bacteria from yogurt on enzyme and serum cholesterol levels of experimentally induced hyperlipidemic Wistar Albino rats. Am. J. Biol. Life Sci. 4:48.

Satil, F., Azcan, N., and Baser, K. (2003). Fatty acid composition of pistachio nuts in Turkey. Chem. Nat. Compounds 39, 322-324. doi: 10.1023/B:CONC.0000003408.63300.b5

Sato, B., Nakajima, H., Fujita, T., Takase, S., Yoshimura, S., Kinoshita, T., et al. (2005). FR177391, A new anti-hyperlipidemic agent from serratia. J. Antibiot. 58, 634. doi: 10.1038/ja.2005.87

Schloss, P. D., Westcott, S. L., Ryabin, T., Hall, J. R., Hartmann, M., Hollister, E. B., et al. (2009). Introducing mothur: open-source, platformindependent, community-supported software for describing and comparing microbial communities. Appl. Environ. Microbiol. 75, 7537-7541. doi: 10.1128/AEM.01541-09

Shih, K.-C., Kwok, C.-F., Hwu, C.-M., Hsiao, L.-C., Li, S.-H., Liu, Y.-F., et al. (1997). Acipimox attenuates hypertriglyceridemia in dyslipidemic noninsulin dependent diabetes mellitus patients without perturbation of insulin sensitivity and glycemic control. Diabetes Res. Clin. Pract. 36, 113-119. doi: 10.1016/S0168-8227(97)00039-9

Tamburini, S., and Clemente, J. C. (2016). Zooming in on inflammatory bowel disease: microbial and proteomic features associated With IBD in 
colonic micro environments. Cell. Mol. Gastroenterol. Hepatol. 2, 540-541. doi: 10.1016/j.jcmgh.2016.07.001

Tandy, S., Chung, R. W., Wat, E., Kamili, A., Berge, K., Griinari, M., et al. (2009). Dietary krill oil supplementation reduces hepatic steatosis, glycemia, and hypercholesterolemia in high-fat-fed mice. J. Agric. Food Chem. 57, 9339-9345. doi: $10.1021 /$ jf 9016042

Tomasello, G., Tralongo, P., Damiani, P., Sinagra, E., Di, T. B., Zeenny, M. N., et al. (2011). Dismicrobism in inflammatory bowel disease and colorectal cancer: changes in response of colocytes. World J. Gastroenterol. 20, 18121-18130. doi: 10.3748/wjg.v20.i48.18121

Tuovinen, E., Keto, J., Nikkilä, J., Mättö, J., and Lähteenmäki, K. (2013). Cytokine response of human mononuclear cells induced by intestinal Clostridium species. Anaerobe 19, 70-76. doi: 10.1016/j.anaerobe.2012.11.002

Turnbaugh, P. J., Ley, R. E., Mahowald, M. A., Magrini, V., Mardis, E. R., and Gordon, J. I. (2006). An obesity-associated gut microbiome with increased capacity for energy harvest. Nature 444, 1027-1131. doi: 10.1038/nature05414

Tyler, A. D., Knox, N., Kabakchiev, B., Milgrom, R., Kirsch, R., Cohen, Z., et al. (2013). Characterization of the gut-associated microbiome in inflammatory pouch complications following ileal pouch-anal anastomosis. PLoS ONE 8:e66934. doi: 10.1371/journal.pone.0066934

Wang, Q., Garrity, G. M., Tiedje, J. M., and Cole, J. R. (2007). Naïve Bayesian classifier for rapid assignment of rRNA sequences into the new bacterial taxonomy. Appl. Environ. Microbiol. 73, 5261-5267. doi: 10.1128/AEM.00062-07
Yu, Z., and Morrison, M. (2004). Improved extraction of PCR-quality community DNA from digesta and fecal samples. BioTechniques 36, 808-812.

Zhang, C., Zhang, M., Wang, S., Han, R., Cao, Y., Hua, W., et al. (2010). Interactions between gut microbiota, host genetics and diet relevant to development of metabolic syndromes in mice. ISME J. 4, 232-241. doi: 10.1038/ismej.2009.112

Zhang, X., Zhao, Y., Xu, J., Xue, Z., Zhang, M., Pang, X., et al. (2015). Modulation of gut microbiota by berberine and metformin during the treatment of high-fat diet-induced obesity in rats. Sci. Rep. 5:14405. doi: 10.1038/srep14405

Zhang, X., Zhao, Y., Zhang, M., Pang, X., Xu, J., Kang, C., et al. (2012). Structural changes of gut microbiota during berberine-mediated prevention of obesity and insulin resistance in high-fat diet-fed rats. PLoS ONE 7:e42529. doi: 10.1371 /journal.pone. 0042529

Conflict of Interest Statement: The authors declare that the research was conducted in the absence of any commercial or financial relationships that could be construed as a potential conflict of interest.

Copyright $\odot 2017 \mathrm{Lu}$, Sun, Li, Zhang, Zhou and Su. This is an open-access article distributed under the terms of the Creative Commons Attribution License (CC BY).

The use, distribution or reproduction in other forums is permitted, provided the original author(s) or licensor are credited and that the original publication in this journal is cited, in accordance with accepted academic practice. No use, distribution or reproduction is permitted which does not comply with these terms. 